\title{
Metástase Gástrica de Câncer de Mama: Relato de Caso e Revisão de Literatura
}

\author{
Gastric Metastases in Primary Breast Cancer: Case Report and Literature Review
}

\author{
Luiz Henrique de Lima Araújo', Andréia Cristina de Melo', Melba Moura Lobo Moreira', Cleudes Alice Sousa Gomes², Hélio Noronha Júnior², \\ Walter Marcos Luiz da Cunha ${ }^{3}$, Maria de Fátima Dias Gauín
}

\section{Resumo}

Introdução: O câncer de mama é a neoplasia mais incidente no sexo feminino com 48.930 novos casos estimados em 2006, no Brasil. Os principais sítios de metástases são: ossos, pele, linfonodos, pulmões, pleura, fígado e cérebro. Metástases gástricas são raras e pouco citadas na literatura, sendo o câncer de mama o segundo principal responsável. À endoscopia digestiva alta, apresentam-se mais comumente sob a forma de infiltração intramural difusa, assemelhando-se à linite plástica, e predominam em tumores do tipo lobular. A comparação histológica dos espécimes é obrigatória, porém a imunoistoquímica também pode ser útil. O prognóstico é ruim, visto que a doença metastática raramente se restringe ao estômago. $\mathrm{O}$ tratamento é paliativo e inclui quimioterapia, terapia hormonal e radioterapia. Relato de caso: Paciente com câncer de mama em tratamento adjuvante com anastrozol apresentou-se ao serviço com queixa de plenitude gástrica e emagrecimento. Havia massa endurecida em epigástrio, dolorosa à palpação profunda. Ao exame endoscópico, observou-se lesão infiltrante, comprometendo o fundo e metade proximal do corpo gástrico. Foi verificado carcinoma ductal com áreas secretoras de muco e células em "anel de sinete" metastático para o estômago; CK7 positivo, CK20 e receptor hormonal negativos. Sem evidência de doença em outros focos, a paciente foi tratada com seis ciclos de docetaxel, obtendo-se resposta patológica gástrica completa. Discussão: Apesar de rara, a metástase gástrica é causa de considerável morbidade em pacientes com câncer de mama e pode responder ao tratamento sistêmico apropriado.

Palavras-chave: Câncer de Mama, Metástase Gástrica, Docetaxel

\footnotetext{
${ }^{1}$ Programa de Residência Médica em Oncologia Clínica do Instituto Nacional de Câncer (INCA) - Rio de Janeiro (RJ), Brasil ${ }^{2}$ Departamento de Oncologia Clínica do Hospital de Câncer III - Instituto Nacional de Câncer (INCA) - Rio de Janeiro (RJ), Brasil ${ }^{3}$ Serviço de Patologia do Instituto Nacional de Câncer (INCA) - Rio de Janeiro (RJ), Brasil

Endereço para correspondência: Luiz Henrique de Lima Araújo. Rua Washington Luís, 85 ap. 601 - Centro - Rio de Janeiro (RJ), Brasil CEP: 20230-026. E-mail: luizmed@uol.com.br
} 


\section{INTRODUÇÃO}

O câncer de mama é a neoplasia maligna mais incidente no sexo feminino, excluindo o câncer de pele não-melanoma, e a segunda principal causa de morte por câncer em mulheres nos Estados Unidos ${ }^{1}$. Para 2007, a Sociedade Americana de Câncer estima que 180.480 mulheres terão diagnóstico de câncer de mama e que 40.460 mulheres morrerão de câncer de mama metastático ${ }^{1}$. A incidência vem aumentando nos últimos anos, o que parece ser devido ao uso da terapia de reposição hormonal na pós-menopausa e ao aumento da prevalência da obesidade ${ }^{1}$. No Brasil, o câncer de mama é o mais incidente no sexo feminino, com 48.930 novos casos estimados em 2006 2 . Apesar dos avanços na triagem, na cirurgia, na radioterapia, na terapia hormonal e na quimioterapia em estágios precoces, a doença metastática permanece incurável e a sobrevida mediana após descoberta de metástases ainda é de apenas dois a três anos ${ }^{3}$. Os principais sítios de metástases por câncer de mama são: ossos, pele, linfonodos, pulmões, pleura, fígado e cérebro ${ }^{3}$.

Metástases gástricas são raras e pouco citadas na literatura. $\mathrm{O}$ câncer de mama é o segundo principal sítio primário, ficando atrás apenas do melanoma maligno ${ }^{4}$. $\mathrm{O}$ acometimento gástrico é reconhecido em $6 \%$ das pacientes com câncer de mama ao longo da vida ${ }^{5}$, porém séries de autopsias demonstram freqüência de até $18 \%{ }^{6}$. Estes dados são provavelmente subestimados, uma vez que os sintomas são inespecíficos e se confundem com os efeitos adversos do tratamento ${ }^{7}$. As queixas mais comuns são náuseas e vômitos, dor epigástrica, anorexia, disfagia, pirose $\mathrm{e}$, mais raramente, melena ${ }^{7}$.

A principal modalidade para o diagnóstico correto é a endoscopia digestiva alta (EDA) com biópsia ${ }^{8}$. As metástases gástricas se apresentam mais comumente sob a forma de infiltração intramural difusa, assemelhandose à linite plástica, porém podem também se desenvolver como lesōes nodulares e ulceradas ${ }^{7}$. Há um predomínio de tumor primário do tipo lobular, principalmente associado a lesōes gástricas difusas ${ }^{7-8}$.

A comparação histológica do espécime gástrico e mamário é obrigatória em todos os casos para distinguir metástase de tumor primário do estômago ${ }^{8}$. A imunoistoquímica, incluindo pesquisa de receptores hormonais e citoqueratinas, também pode ser útil ${ }^{8}$. CK20 é uma citoqueratina de baixo peso molecular normalmente expressa no epitélio gastrintestinal, epitélio das vias urinárias e células de Merkell ${ }^{9}$. CK7 é encontrada em tumores de pulmão, ovário, endométrio e mama, estando ausente em tumores do trato gastrintestinal inferior9. O fenótipo CK20+/CK7- sugere fortemente tumores primários do cólon, estando presente em 75\% a 95\% destes. Tumores CK20-/CK7+ podem ter origem em pulmão, mama, trato biliar, pâncreas, ovário e carcinomas de endométrio?.

O prognóstico dos pacientes com metástases gástricas por câncer de mama é muito ruim, visto que a doença metastática raramente se restringe ao estômago ${ }^{5}$. $\mathrm{O}$ tratamento geralmente inclui quimioterapia, terapia hormonal e/ou radioterapia, com intuito paliativo5. A cirurgia é reservada para pacientes com complicaçôes, como perfuração, sangramento maciço ou obstruçãos. Existem poucos casos relatados de tratamento operatório em pacientes com metástase gástrica isolada e este tratamento parece não interferir na sobrevida global ${ }^{10}$.

McLemore et al. revisaram 73 casos de pacientes com câncer de mama metastático para o trato gastrintestinal e/ou peritônio avaliados na Clínica Mayo, no período de 1985 a $2000^{11}$. Todos os casos haviam sido confirmados por análise histológica. $\mathrm{O}$ tratamento cirúrgico paliativo foi realizado em $64 \%$ dos pacientes e não apresentou efeito estatisticamente significativo sobre a sobrevida ( 28 meses versus 26 meses; $\mathrm{p}=0,31)^{11}$. Entre os pacientes com metástases isoladas para o trato gastrintestinal, a sobrevida global após ressecção paliativa foi de 44 meses, comparada a 9 meses no grupo que não foi operado $(\mathrm{p}=0,1)^{11}$.

Em uma série holandesa de 27 pacientes com metástases gástricas de câncer de mama, Taal et al. demonstraram que $93 \%$ das pacientes tinham metástases em outros sítios além do estômago ${ }^{7}$. Nesta mesma série, a sobrevida mediana foi de quase um ano e $22 \%$ das pacientes viveram por mais de dois anos após o diagnóstico das metástases gástricas ${ }^{7}$. Utilizando vários regimes de tratamento, foi descrita taxa de resposta global de $32 \% \%^{7}$. Posteriormente, em nova análise incluindo 51 pacientes com diagnóstico endoscópico de metástases gástricas, os autores encontraram sobrevida mediana de 10 meses, com taxa de sobrevida de $23 \%$ em dois anos ${ }^{12}$. A maioria dos pacientes (65\%) recebeu tratamento sistêmico com quimioterapia ou terapia hormonal e $46 \%$ obtiveram resposta parcial $^{12}$.

\section{RELATO DE CASO}

Paciente de 57 anos, sexo feminino, teve diagnóstico em 2000 de carcinoma ductal invasivo (CDI) em mama direita, estágio IIIB, com pesquisa de receptores hormonais positiva. Após quatro ciclos de quimioterapia 
neo-adjuvante com AC [doxorrubicina $60 \mathrm{mg}$ por metro quadrado de área de superfície corporal $\left(\mathrm{mg} / \mathrm{m}^{2}\right.$ ASC) e ciclofosfamida $600 \mathrm{mg} / \mathrm{m}^{2}$ ASC intravenosos a cada 21 dias], foi submetida à mastectomia radical modificada (MRM), com tumor de $4 \mathrm{~cm} \times 2 \mathrm{~cm}$ e acometimento metastático em seis linfonodos axilares. Em seguida, recebeu quimioterapia adjuvante com um ciclo de $\mathrm{AC}$ e cinco ciclos de CMF clássico (ciclofosfamida oral $100 \mathrm{mg} / \mathrm{m}^{2} / \mathrm{dia}$ ASC por 14 dias, metotrexate intravenoso $40 \mathrm{mg} / \mathrm{m}^{2}$ ASC nos dias 1 e 8 e fluorouracil intravenoso $600 \mathrm{mg} / \mathrm{m}^{2}$ ASC nos dias 1 e 8 em ciclos de 28 dias) e radioterapia adjuvante na dose de 50Gy em 25 frações. Fez uso de tamoxifeno na dose de $20 \mathrm{mg}$ por dia no período de janeiro de 2002 a dezembro de 2003, quando foi diagnosticado CDI metacrônico em mama esquerda, estágio IIIB, com pesquisa negativa para receptores hormonais. Após três ciclos de FAC (fluorouracil $500 \mathrm{mg} / \mathrm{m}^{2}$ ASC, doxorrubicina $50 \mathrm{mg} / \mathrm{m}^{2}$ ASC e ciclofosfamida $500 \mathrm{mg} / \mathrm{m}^{2}$ ASC intravenosos a cada 21 dias) neoadjuvante, foi realizada MRM, com tumor de $2 \mathrm{~cm}$ e cinco linfonodos axilares acometidos por CDI com áreas secretoras de muco (Figura 1). Fez tratamento adjuvante com CMF clássico e foi iniciado anastrozol na dose de $1 \mathrm{mg}$ por dia, em outubro de 2004.

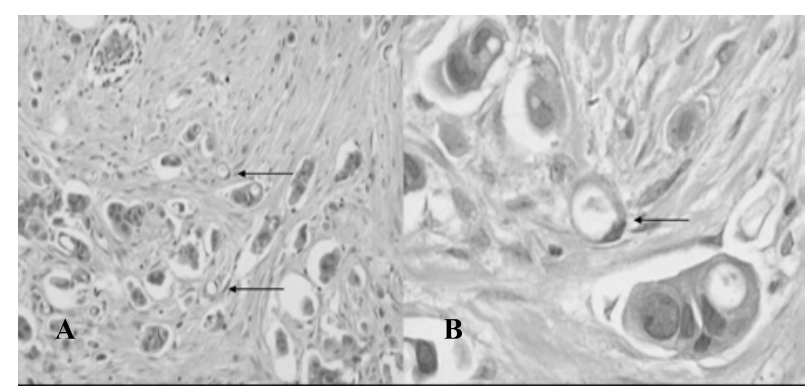

Figura 1. A - Carcinoma ductal infiltrante com células em "ane de sinete" (HE). B - Detalhe de célula em "anel de sinete" (HE)

Em fevereiro de 2006, apresentou quadro de plenitude gástrica e emagrecimento. Apresentava-se hipocorada +/4+, hidratada, eutrófica, anictérica e sem edemas. O exame do aparelho cardiorrespiratório era normal. Ao exame do abdome, havia massa endurecida em epigástrio, dolorosa à palpação profunda. A revisão laboratorial demonstrou leve anemia, com nível sérico de hemoglobina de $11,4 \mathrm{~g} / \mathrm{dL}$ e hematócrito de $34 \%$, sem alterações às provas de função hepática e renal. Submetida à EDA, foi visualizada lesão infiltrante comprometendo o fundo e metade proximal do corpo gástrico. Ao exame histológico, verificou-se carcinoma ductal com áreas secretoras de muco e células em "anel de sinete" metastático para o estômago (Figura 2A). A imunoistoquímica revelou positividade para citoqueratina 7 (CK7) (Figura 2B) e negatividade para CK20 e receptores hormonais. Sem outros focos de doença, foram oferecidos seis ciclos de quimioterapia com docetaxel $\left(75 \mathrm{mg} / \mathrm{m}^{2}\right.$ ASC intravenoso a cada 21 dias). Durante o tratamento, observou-se melhora clínica, ganho de peso e redução da massa abdominal. À EDA de controle, havia apenas hipertrofia do pregueado mucoso da grande curvatura no corpo gástrico. A análise histológica dos espécimes de biópsia revelou mucosa gástrica superficial livre de neoplasia, apresentando gastrite crônica leve sem metaplasia intestinal. A paciente encontra-se atualmente em acompanhamento pela oncologia clínica, realizando exames de reestadiamento, tendo sido demonstrada progressão de doença para ossos e fígado em janeiro de 2007.

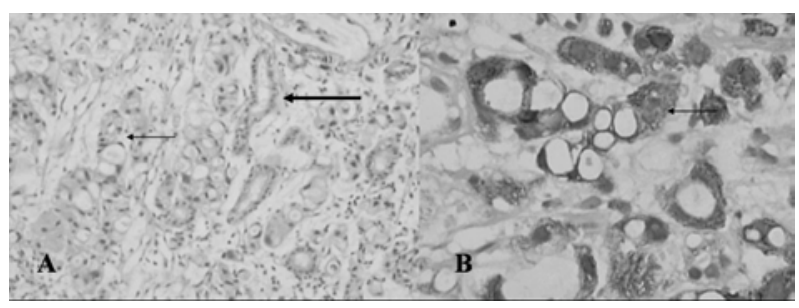

Figura 2. A - Carcinoma ductal infiltrante metastático (seta pequena) para mucosa gástrica (seta grande) (HE). B - Imunoistoquímica: positividade ao CK7 (seta)

\section{DISCUSSÃO}

No caso relatado, a paciente se apresentou com quadro de plenitude gástrica, emagrecimento, anemia e massa abdominal, achados que motivaram maior propedêutica. À EDA, havia infiltração comprometendo o fundo e metade proximal do corpo gástrico. $\mathrm{O}$ exame histológico permitiu afirmar o diagnóstico de adenocarcinoma metastático de mama através de comparação direta entre os materiais obtidos de biópsia gástrica e mamária, assim como pela observação de semelhanças entre esses tumores pela imunoistoquímica.

À semelhança do carcinoma metacrônico em mama esquerda, as metástases gástricas tinham pesquisa negativa para receptores hormonais. O fenótipo CK20/CK7+ é compatível com a origem mamária. Apesar de muito mais prevalente, os carcinomas ductais invasivos raramente estão envolvidos com metástases para o trato gastrintestinal ${ }^{8}$. O caso relatado traz esta associação como uma de suas particularidades. Chama também a atenção pela ausência de outros focos de doença metastática, 
bem como pelo controle da doença com tratamento quimioterápico, não sendo demonstrada lesão residual após EDA de controle com biópsias. Apesar de a EDA poder gerar falso-negativos em até $30 \%$ das pacientes com metástases gástricas, provavelmente devido à infiltração de células tumorais localizada em camadas mais profundas em relação à mucosa, a EDA e/ou tomografia computadorizada são os exames mais utilizados no acompanhamento ${ }^{12}$. A ausência de alterações pela análise endoscópica e histológica sugere resposta completa ao tratamento.

Apesar de rara, a metástase gástrica é causa de considerável morbidade em pacientes com câncer de mama e pode responder a tratamento sistêmico apropriado. Deve ser considerada no diagnóstico diferencial de pacientes oncológicos com queixas dispépticas, principalmente se persistentes e uma vez excluídas causas mais comuns, como doença cloridopéptica, hipercalcemia, metástases hepáticas e efeitos adversos do tratamento.

\section{REFERÊNCIAS}

1. Jemal A, Siegel R, Ward E, Murray T, Xu J, Thun MJ, et al. Cancer statistics 2007. CA Cancer J Clin. 2007;57:43-66.

2. Instituto Nacional de Câncer / Ministério da Saúde. Estimativa 2006: incidência de câncer no Brasil. Rio de Janeiro: INCA. [acesso em mar 2007]. Disponível em: $<$ http://www.inca.gov.br>

3. Wood WC, Muss HB, Solin LJ, Olopade OI. Malignant tumors of the breast. In: De Vita Jr VT, Hellman S,
Rosenberg SA. Cancer: principles and practice of oncology. 7th ed. Philadelphia: Lippincott Williams \& Wilkins, 2005:32:1415-477.

4. Bognel C, Lasser P, Zimmermann P. Gastric metastases: apropos of 17 cases [in French]. Ann Chir. 1992;46:436-41.

5. Amicucci G, Sozio ML, Sozio A, Rizzo FM. Gastric metastases of breast carcinoma [Letter]. Am J Gastroenterol. 1999;94:859.

6. Rimlinger H, Soulard R, Romand F, Rapp C, Dubourg P, Berets $\mathrm{O}$. Métastase gastrique du cancer du sein. Presse Med. 2001;30(3):634-35.

7. Taal BG, den Hartog JFC, Steinmetz R, Peterse H. The spectrum of gastrointestinal metastases of breast carcinoma I: stomach. Gastrointest Endosc. 1992;38:130-35.

8. Schwarz RE, Klimstra DS, Turnbull ADM. Metastatic breast cancer masquerading as gastrointestinal primary. Am J Gastroenterol. 1998;93(1):111-14.

9. Varadhachary GR, Abbruzzese JL, Lenzi R. Diagnostic strategies for unknown primary cancer. Cancer. 2004;100(9):1776-785.

10. Zelek L, Cottu PH, Mignot L, Roquancourt A, Fizazi K, Cojean-Zelek I, et al. Gastric metastases from breast cancer: a retrospective series of 12 patients. Am J Clin Oncol. 2001;24(4):363-65.

11. McLemore EC, Pockaj BA, Reynolds C, Gray RJ, Hernandez JL, Grant CS, et al. Breast cancer: presentation and intervention in women with gastrointestinal metastasis and carcinomatosis. Ann Surg Oncol. 2005;12(11):886-94.

12. Taal BG, Peterse H, Boot H. Clinical presentation, endoscopic features, and treatment of gastric metastases from breast carcinoma. Cancer. 2000;89:2214-221.

\section{Abstract}

Introduction: Breast cancer is the most common neoplasm in women. In Brazil, approximately 48,930 new cases are expected in the year 2006. Sites for metastases from primary breast cancer include lymph nodes, bones, bone marrow, lungs, liver, and central nervous system. Reports of gastric metastasis are rare in published series on advanced breast cancer. Upper gastrointestinal endoscopy is usually performed and shows a diffuse intramural infiltration similar to linitis plastica, found most commonly in cases presenting lobular carcinoma. A comparative histopathological study of mammary and gastrointestinal specimens is mandatory, but immunohistochemical techniques may also be useful. The prognosis is often reserved. Treatment is palliative, including systemic chemotherapy, hormonal therapy, and radiation therapy. Case report: A patient in adjuvant therapy for breast cancer with anastrozole presented with epigastric pain and weight loss. Physical examination revealed a palpable epigastric mass, and upper gastrointestinal endoscopy revealed an infiltrating lesion located at the antrum and corpus of the stomach. Histopathological examination confirmed metastatic ductal carcinoma with mucosecretory areas and "signet ring" cells (CK7-positive and CK20 and hormone receptor-negative). Since there were no other disease sites, the patient was treated with six cycles of docetaxel, leading to a complete gastric pathological response. Discussion: Although rare, gastric metastasis can involve high morbidity in patients with breast cancer and may respond to appropriate systemic antineoplastic therapy.

Key words: Breast cancer, Gastric metastasis, Docetaxel 